\title{
Prevalence of micro and macrovascular complications of diabetes detected at single visit screening
}

\author{
B M A C Balasuriya ${ }^{1}$, M R Sumanatilleke ${ }^{2}$, T I Jayasekera ${ }^{3}$, M A Wijesuriya ${ }^{4}$, N P Somasundaram ${ }^{5}$
}

Sri Lanka Journal of Diabetes, Endocrinology and Metabolism 2012; 2: 17-20

(Index words: complications, diabetes, prevalance)

\begin{abstract}
Objective: To describe the prevalence of complications of diabetes and to study the relationship between the prevalence of complications and the duration of diabetes in a subset of Sri Lankan population

Study design: Cross sectional case analysis of 6765 patients at National Diabetes Centre, Rajagiriya, Sri Lanka.

Data analysis: Data was analyzed with SPSS 11.

Results: The mean duration of diabetes in the study population was 7.9 years $(\mathrm{SD}=6.7)$. The results showed that the prevalence of micro vascular complications increased with increasing duration of diabetes. In patients with diabetes mellitus for less than 1 year, retinopathy prevalence was $6.8 \%$ and in those with 16-20 years diabetes duration it rose to $52.6 \%$. Neuropathy prevalence at diagnosis was $11 \%$ and at $16-$ 20 years it was $54.3 \%$. Nephropathy prevalence at diagnosis and at $16-20$ years was $18.8 \%$ and $23.8 \%$ respectively.
\end{abstract}

Conclusions: The study has demonstrated high prevalence of complications at the initial diagnosis. Hence, early screening techniques would be beneficial in order to prevent and retard the progression of the disease and to reduce the associated morbidity and mortality.

\section{Introduction}

Prevalence of diabetes mellitus particularly type 2 has risen steadily over the past few decades globally. People with diabetes have an increased risk of developing microvascular complications like diabetic retinopathy, diabetic nephropathy and diabetic neuropathy, which, if undetected or left untreated, can have a devastating impact on quality of life and place a significant burden on health care costs.

Diabetic microvascular complications can reduce life expectancy. The strongest risk factors are poor glycaemic control and duration of diabetes. Other modifiable risk factors such as hypertension, dyslipidaemia smoking, as well as non-modifiable risk factors such as age at onset of diabetes and genetic factors all contribute to the progression of complications. Duration of diabetes and poor glycaemic control has been identified as the main factors leading to the development of complications (4).

Evidence is accumulating that complications are common among South Asian diabetic population including Sri Lankans, particularly in terms of cardiovascular and renal disease.
This study is aimed at estimating the prevalence of major micro and macrovascular complications of diabetes among subjects who presented for single visit screening at the National Diabetes Centre, Rajagiriya. We also studied any association between the duration of the disease and prevalence of complications.

\section{Methods}

\section{Population characteristics}

Study sample consisted of 6765 patients (4121 males and 2629 females) who had at least two screening visits to the National Diabetes Centre at Rajagiriya. Both type 1 and type 2 diabetes patients were included. The mean age of the study group was 51.63 years range. There were $95.5 \%$ patients with type 2 diabetes and $4.5 \%$ with type 1 diabetes. Study sample represented $14.1 \%$ rural and $85.7 \%$ urban dwellers. Employment categorisation breakdown shows $46.5 \%$ employed (12.9\% professionals and 33.6 non professionals), 9.5 retired and $43.1 \%$ unemployed people in the study sample.

${ }^{1}$ Consultant Physician, General Hospital Nuwaraeliya, ${ }^{2}$ Consultant Endocrinologist, Teaching Hospital, Karapitiya, ${ }^{3}$ Medical Officer, National Diabetes Centre, ${ }^{4}$ President, National Diabetes Centre, ${ }^{5}$ Endocrinologist, National Hospital of Sri Lanka, Colombo. 


\section{Methodology}

Electronic medical records of patient data were analysed and results were categorised into major micro and macrovascular complications of diabetes. Screening for diabetic retinopathy was carried out by dilated fundoscopy and a retinal camera (Topcon). All grades of diabetic retinopathy ranging from background retinopathy up to vitreous haemorrhage, in one or both eyes were included as diabetic retinopathy. Diabetic nephropathy included patients who were already diagnosed as having diabetic nephropathy by a physician or those with microalbuminuria above $20 \mathrm{~g} / \mathrm{dl}$ on early morning spot urine sample (analysed by Daytona analyser) in the presence of a blood glucose level $<200 \mathrm{mg} / \mathrm{dl}$ and a normal urine full report. Creatinine clearance was calculated by using the Cockcroft Gault formula. Those with nephropathy were further subcategorised into stages of CKD based on estimated GFR.

Patients were considered as having diabetic neuropathy when the clinical examination by a trained medical officer at the National Diabetes Centre showed that the sensory modalities of touch, vibration and pain were either impaired or absent in one or both feet. Touch was tested using cotton wool, pressure was tested with $10 \mathrm{~g}$ monofilament (Semmes Weinstein) and vibration was tested by using $128 \mathrm{~Hz}$ tuning fork.

Patients were diagnosed as having ischaemic heart disease if they carried a diagnosis. Data on stroke was not systematically captured and hence not analysed in this study. Diagnosis of peripheral vascular disease was made when the ankle brachial pressure index was $<0.8$. Blood pressure was checked with a standard manual sphygmomanometer and where the pedal pulse was weak or absent Doppler study was used to detect the blood flow in lower limb arteries.

We categorised the duration of diabetes into 6 groups as follows, Group1 (<1 year), Group 2 (1-5 years ), Group
3 (6 - 10 years), Group 4 (11 - 15 years), Group 5 (16 - 20 years), Group 6 (> 20 years). There were 690 (10\%) subjects in Group 1, 2353 (34.8\%) in Group 2, 1710 (25.3\%) in Group 3, 1034 (15.2\%) in Group 4, 689 (10\%) in Group 5, 289 (4.2\%) in Group 6.

\section{Data analysis}

We analysed the data using SPSS version 11.

\section{Results}

The mean duration of diabetes in the study population was 7.9 years $(\mathrm{SD}=6.7)$. There were $95.5 \%$ patients with type $2 \mathrm{DM}$ and $4.5 \%$ with type $1 \mathrm{DM}$.

Majority of retinopathy cases were among the type 2 DM patients. Our results show a progressive rise in the number of retinopathy cases as the duration of diabetes increased (Table 1). There were 6.8\% $(n=49)$ in < 1year group, $28.3 \%(n=489)<10$ year group, while in those patients with diabetes of $>20$ years the figure rose up to $57.8 \%(n=175)$. Even severe degrees of retinopathy was noted among patients with duration of diabetes $<1$ year. There were $6.8 \%$ patients with established retinopathy and among them 11 (23.3\%) had background retinopathy, while 3 (6.2\%) had evidence of maculopathy in $<1$ year group. Diabetic neuropathy also demonstrated a similar trend with $<1$ year group showing a prevalence of $11 \%, 6$ - 10 year group $28.6 \%$ and over 20 year group rising to a figure of $65.3 \%$. Table 1 summarizes the prevalence of microvascular complications in each group of patients with diabetes mellitus.

The prevalence of nephropathy (microalbuminuria) in $<1$ year group was $18.8 \%$, in 6 -10 year group $21.2 \%$ and in the over 20 year group $25.4 \%$. Those with nephropathy were further subcategorised into stages of CKD based on estimated GFR using the Cockcroft Gault formula. Table 2 shows the prevalence.

Table 1. Prevalence of complications of diabetes mellitus

\begin{tabular}{ccccccc}
\hline Complications & \multicolumn{5}{c}{ Duration of diabetes in years } \\
\cline { 2 - 7 } & $<$ 1year & $1-5$ & $6-10$ & $11-15$ & $16-20$ & $>20$ \\
\hline Retinopathy (\%) & 6.8 & 12.1 & 28.3 & 39.7 & 52.6 & 57.8 \\
Nephropathy (\%) & 18.8 & 19.7 & 21.2 & 19.8 & 23.8 & 25.4 \\
Neuropathy (\%) & 11 & 16.7 & 28.6 & 40 & 54.3 & 65.3 \\
\hline
\end{tabular}


Table 2. CKD staging of patients who had nephropathy

\begin{tabular}{lccccc}
\hline Duration (years) & Stage 1 (\%) & Stage 2 (\%) & Stage 3 (\%) & Stage 4 (\%) & Stage 5 (\%) \\
\hline $0-1$ & 6.2 & 43.3 & 13.6 & 0.4 & 0.3 \\
$1-5$ & 5.8 & 45.3 & 17.9 & 0.8 & 0 \\
$6-10$ & 3.1 & 47.4 & 25.7 & 0.8 & 0.1 \\
$11-15$ & 2.6 & 47.4 & 32.8 & 0.6 & 0.1 \\
$16-20$ & 2.8 & 43.2 & 37.9 & 2.3 & 0.5 \\
\hline
\end{tabular}

Table 3. Comparison of Sri Lankan data with other countries

\begin{tabular}{lccc}
\hline Complication & Sri Lanka & India & Korea \\
\hline Retinopathy & 25.7 & 23.7 & 47.8 \\
Neuropathy & 28.4 & 27.5 & 31.9 \\
Nephropathy & 20.4 & 5.5 & 41 \\
PVD & 0.5 & 4 & 0.4 \\
HDD & 5.6 & 11.4 & 2.4 \\
Hypertension & 60.7 & 38 & - \\
\hline
\end{tabular}

Diabetic peripheral neuropathy was observed in 11\% $(n=79)$ patients $<1$ year group, 28.6\% $(n=493)$ in $6-10$ year group, and in $54.3 \%(n=344)$ in 16 - 20 year group (Table 1). Neuropathy was diagnosed if atleast one of the above mentioned criteriae were present in one or both feet (abscense of vibration, touch or positive monofilament test $->3 / 10)$.

Peripheral vascular disease was found in 7 patients $(0.1 \%)$ in the $<1$ year group, $7(0.1 \%)$ in 6 - 10 year group, and in $88(1.3 \%)$ in the 16 - 20 year group. The prevalence of IHD was $2.2 \%(n=16)$ in <year group, $5 \%$ in 6 - 10 years and $10.6 \%$ in 16 - 20 years. Hypertension was detected in 4106 (60.7\%) patients.

\section{Discussion}

The chronic complications of diabetes mellitus are important prognostic factors in diabetes and also reflect the standards of care the patients receive. The pathogenic mechanisms are variable and differ according to the race. In general the development of diabetic microangiopathy depends on the duration and the severity of disease while that of macro angiopathy does not. Age and duration of diabetes have been the most frequently mentioned risk factors for new onset type 2 diabetes complications $(3,4)$. Harris (4) has suggested, the presence of diabetic complications in type $2 \mathrm{DM}$ may be a consequence of untreated hyperglycaemia or other factor while diabetes remain clinically undiagnosed (4). He has also shown that $50 \%$ type 2 DM patients in Europe had presence of complications at time of diagnosis. As the incidence of diabetes continues to rise, the burden of diabetic microvascular complications will also increase. To reduce the associated morbidity and mortality it is essential that factors associated with the onset and progression of diabetes-related complications are identified as early as possible.

In our study, we have shown that, even during the first year of diagnosis the prevalence of diabetic retinopathy was $6.8 \%$ and diabetic nephropathy prevalence during the first 5 years was as high as $64 \%$. A significant proportion (45\%) had evidence of stage 2 diabetic nephropathy during the first year of detection of diabetes. Table 3 compares the results of our study with results from regional countries like India (2) and Korea (1). The prevalence of retinopathy and neuropathy in Sri Lanka is comparable to those in India. However, our figure for diabetic nephropathy is about 4 times higher than that of 
India. The studies quoted are very old ones (1999), and it is better to mention what criteria they have used to diagnose each complication and the observed differences for example higher diabetic retinopathy and neuropaty in Korea is due to this effect.

Randomised intervention trials have shown that intensive treatment (tight control of blood glucose, blood pressure and lipids) delays the onset and slows the progression not only of diabetic retinopathy, but also of diabetic nephropathy in patients with type 1 diabetes (7). In the Diabetes Control Complications Trial intensive therapy reduced the mean adjusted risk of the cumulative incidence of microalbuminuria in the primary prevention cohort by $34 \%$ and the albumin excretion rate by $15 \%$ following the first year of therapy.

One of the shortcomings of our study is that we did not screen patients routinely for asymptomatic ischaemic heart disease which is common in diabetes mellitus.

We have been able to demonstrate a considerable degree of retinopathy and nephropathy affects the local population within the first year of diagnosis. These findings should be an eye opener to both the clinician as well as the health care planners of the country. Because of the chronic nature, insidious complications, and the means required to control diabetes being costly, diabetes mellitus is a major challenge to the health system. The therapeutic implications are that early detection and effective screening programme is mandatory in this regard as Sri Lanka is already witnessing an upsurge of diabetes.

\section{References}

1. Nam JH, Lee SH, Lee HJ, Han JH, et al. Overall prevalence of diabetic retinopathy in urban Korean patients. Korean Medical Journal 1999; 23(5): 702-14.

2. Ramachandran A, Snehalatha C, Latha E, Manoharan M, Vijay. Diabetic retinopathy at the time of diagnosis in NIDDM patients. Diab Res Clin Prac 1999: 44; 207 - 13.

3. Petersen JL, McGuire DK. Impaired glucose tolerance and impaired fasting glucose - a review of diagnosis, clinical implications and management. Diab Vasc Dis Res 2005; 2(1): 9 - 15 .

4. Harris MI, Klein R, Welborn TA, Knutman MW. Onset of NIDDM occurs at least 4- 7 years before clinical diagnosis. Diabetes Care 1992; 15: 815-9.

5. Pinhas-Hamiel O, Zeitler P. The global spread of type 2 diabetes mellitus in children and adolescents. J Pediatr 2005; 146: 693.

6. Flegal MI, Cowie CC, et al. Prevalence of diabetes, impaired fasting glucose, and impaired glucose tolerance in US adults. Diabetes Care 1998; 21(4): 518 - 24.

7. The Diabetes Control and Complications Trial Research Group. The effect of intensive treatment of diabetes on the development and progression of long term complications in insulin-dependent diabetes mellitus. N Engl J Med 1993; 329: 977 - 86.

8. Girach A, Manner D, Porta M. Diabetic microvascular complications: a review. International Journal of Clinical Practice 2006; 60(11): 1471-83.

9. Finne P, Reunanen A, Stenman S, et al. Incidence of endstage renal disease in patients with type 1 diabetes. JAMA 2005; 294: 1782. 